\title{
Plantas medicinais cultivadas em quintais de comunidades rurais no domínio do cerrado piauiense (Município de Demerval Lobão, Piauí, Brasil)
}

AGUIAR, L.C.G.G.; BARROS, R.F.M.*

Departamento de Biologia, UFPI, Avenida Universitária, 1310, Bairro Ininga, CEP: 64.049-550, Teresina-Brasil

*rbarros.ufpi@yahoo.com.br

RESUMO: O conhecimento terapêutico acumulado por populações rurais através de séculos de estreito contato com o meio possibilita a obtenção de informações acerca do uso dos recursos naturais. O objetivo do presente trabalho foi realizar um inventário das plantas medicinais cultivadas em quintais do município de Demerval Lobão, Piauí, bem como, conhecer o emprego e a importância dessas espécies na comunidade. Foram amostrados 21 quintais em nove comunidades da zona rural do município de Demerval Lobão com vegetação de cerrado. O trabalho foi conduzido com os mantenedores dos quintais a partir de entrevistas semi-estruturadas e observação direta, associada à técnica de turnê-guiada. As plantas medicinais coletadas foram catalogadas, identificadas, e depositadas no Herbário Graziela Barroso (TEPB) da Universidade Federal do Piauí (UFPI). Foram referidas 100 espécies botânicas, predominantemente herbáceas e cultivadas, distribuídas em 49 famílias, sendo Leguminosae, Euphorbiaceae e Lamiaceae as mais representativas em número de espécies. Na preparação dos remédios, as folhas foram as mais utilizadas, sendo a decocção a principal forma de preparo. As doenças mais frequentes tratadas por remédios caseiros referem-se ao sistema respiratório e digestivo. Observou-se que não há rigidez quanto à posologia e a duração do tratamento, ficando este a critério do hábito de cada pessoa entrevistada. As plantas que apresentaram índice de importância relativa e concordância de uso (CUP) acima de 60\% foram cansansão (Cnidosculus urens), boldo (Plectranthus barbatus Andrews), jurema-preta (Mimosa verrucosa Benth) e cidreira (Lippia alba (Mill.) N.E.Br. ex Britton \& P.Wilson). $P$. barbatus foi a mais citada e também a que apresentou os índices mais altos de CUP $(100 \%)$, fator de correção - FC $(1,00)$ e concordância de uso corrigido - CUPc $(100 \%)$ para dores no estômago e problemas no fígado. Observou-se a necessidade de resgatar o conhecimento acerca das plantas medicinais para contribuir na melhoria da qualidade de vida dos moradores da zona rural, como primeiro passo para a valorização e adequação dos recursos da medicina popular para o tratamento de doenças mais frequentes.

Palavras-chave: etnomedicina, índice de importância relativa, concordância de uso, Piauí

ABSTRACT: Medicinal plants cultivated in homegardens of rural communities in the domain of Cerrado in Piaui (Demerval Lobão Municipality, Piauí State, Brazil). Therapeutic knowledge accumulated by rural populations, through centuries of direct contact with the environment, makes possible the gathering of information concerning use of natural resources. The aim of the present study was to carry out an inventory of the medicinal plants cultivated in homegardens of Demerval Lobão Municipality, Piauí State, Brazil, as well as to learn their uses and importance in the community. A total of 21 homegardens in nine communities of the rural area of Demerval Lobão Municipality with Cerrado vegetation were sampled. The study was conducted by means of semi-structured interviews and direct observation, associated with the technique of a tour guided by the keepers of the homegardens. The collected medicinal plants were cataloged, identified and deposited in the Herbarium Graziela Barroso (TEPB) of Federal University of Piauí (UFPI). Of 100 botanical species, predominantly herbaceous and cultivated species distributed in 49 families, were cited, and the most representative families in number of species were Leguminosae, Euphorbiaceae and Lamiaceae. For the preparation of medicines, leaves were most used and decoction was the main form of preparation. The most frequent diseases treated by homemade remedies are associated with the respiratory and digestive systems. There is no rigidity as to dosage and duration of treatment; it depended on the habit of each interviewed

Recebido para publicação em 13/03/2009

Aceito para publicação em 10/04/2012

Rev. Bras. Pl. Med., Botucatu, v.14, n.3, p.419-434, 2012. 
person. The plants that presented an index of relative importance and use agreement above $60 \%$ were the following: "cansansão" (Cnidosculus urens), "boldo" (Plectranthus barbatus Andrews), black-jurema (Mimosa verrucosa Benth) and "cidreira" (Lippia alba (Mill.) N.E.Br. ex Britton \& P.Wilson). P. barbatus was most cited and also the species that presented the highest indices of CUP $(100 \%), F C(1,00)$ and CUPc $(100 \%)$ for stomach pains and liver problems. There is the need of rescuing knowledge concerning medicinal plants to contribute to the improvement of the life quality of the inhabitants of the rural area, as a first step for the appreciation and adaptation of resources of folk medicine for the treatment of the most frequent diseases.

Key words: etnomedicine, index of relative importance, use agreement, Piauí

\section{INTRODUÇÃO}

Em todas as épocas e culturas, o homem aprendeu a tirar proveito dos recursos naturais locais. Ao longo dos anos, argutos observadores perceberam que uma erva capaz de induzir sonolência seria também capaz de acalmar, se usada em dosagens menores. Plantas cujos frutos usualmente tinham efeito laxante poderiam ser usadas com parcimônia para regular intestino preguiçoso. Todo este conhecimento foi passado oralmente ao longo de gerações, que juntamente com mitos e rituais, formaram parte importante das culturas locais (Lorenzi \& Matos, 2002).

Vários autores têm estudado o uso medicinal das plantas e Ming et al. (2002) comentaram a respeito do aumento nesta linha de pesquisa que em todo mundo, e no Brasil, apresenta crescimento visível. Vários motivos levam as pessoas a utilizarem plantas com fins terapêuticos, podendo ser de ordem médica, social, cultural, econômica, ou ainda, filosófica.

Amorozo (1996) definiu doença como evento que desestrutura a ordem cotidiana trazendo sempre consigo insegurança. O uso de plantas na etnomedicina está inserido no contexto social do "homem do campo", uma vez que nas áreas rurais a distância dos postos de saúde é uma barreira que dificulta o acesso aos tratamentos oficiais, sendo o uso dos recursos vegetais na cura de determinadas doenças uma constante.

O quintal é compreendido como um sistema de produção complementar a outras formas de uso da terra e se destaca pelo valor econômico que desempenha na residência, constituindo fonte disponível de recursos alimentícios e medicinais (Pasa et al., 2005). Devese enfatizar, também, a contribuição na conservação da estrutura física e da fertilidade do solo, pois utiliza insumos naturais, como dejetos de animais, restos vegetais, cinzas e terra transportada das matas, a fim de promover o fortalecimento do espaço que é ocupado por uma variedade de plantas (Lok \& Mendez, 1998).

Guarim-Neto \& Morais (2003) fizeram revisão bibliográfica de trabalhos com informações das espécies medicinais do cerrado matogrossense com a intenção de se estabelecer uma base de dados regionais. Essa revisão quantificou 509 espécies, distribuídas em 297 gêneros e 96 famílias. As espécies com maior número de citações bibliográficas foram Stryphnodendron adstringens (Mart.) Coville e Anemopaegma arvense (Vell.) Stelf identificando também que 31\% das espécies eram arbóreas.

Diversos trabalhos etnobotânicos têm retratado o uso de plantas medicinais, tanto nativas como cultivadas, em diferentes regiões brasileiras, como na Amazônia (Amorozo \& Gély, 1988; Sablayrolles, 2004), na Floresta Atlântica (Medeiros et al., 2004; Gazzaneo et al., 2005; Silva \& Andrade, 2005) e em comunidades rurais e urbanas do interior do Brasil (Albuquerque \& Andrade, 2002; Jacoby et al., 2002; Marodin \& Baptista, 2002; Arnous et al., 2005; Pasa et al., 2005). No estado do Piauí, Berg \& Silva (1985), Franco \& Barros (2006) e Santos et al. (2008) desenvolveram estudos com plantas medicinais em comunidades tradicionais no Estado.

O objetivo do presente trabalho foi realizar o levantamento das espécies de plantas medicinais presentes nos quintais e usadas pela população rural do município de Demerval Lobão/PI, bem como, conhecer a finalidade do uso, a parte da planta utilizada, o modo de preparo dos remédios, verificar os valores de uso (VU), a importância relativa (IR) e os índices de concordância de uso das espécies (CUP).

\section{MATERIALE MÉTODO}

\section{Área de estudo}

Criado pela Lei oo 2.553 de 09/12/1963, o município de Demerval Lobão compreende área irregular de $221 \mathrm{~km}^{2}$, com população total de 12.806 habitantes (IBGE, 2008), uma densidade demográfica de $54,79 \mathrm{hab} \mathrm{km}^{-2}$, onde $17,89 \%$ das pessoas residem na zona rural. Quanto à educação, 68,6\% da população acima de 10 anos de idade são alfabetizadas (CEPRO, 2008). A sede municipal localiza-se nas coordenadas geográficas $05^{\circ} 21^{\prime} 28^{\prime \prime}$ S e $42^{\circ} 40^{\prime} 33^{\prime \prime} \mathrm{W}$, distando cerca

Rev. Bras. PI. Med., Botucatu, v.14, n.3, p.419-434, 2012. 
de $30 \mathrm{~km}$ da capital Teresina (IBGE, 2008). A sede do município dispõe de energia elétrica distribuída pela Companhia Energética do Piauí S/A - CEPISA, terminais telefônicos, agência de correios e telégrafos e escolas de ensino fundamental (CEPRO, 2008). Segundo dados do IBGE (2008) a cobertura vegetal é caracterizada pela Floresta Estacional Semidecídua, por áreas com babaçu, pelo Cerrado - formação não florestal, xeromorfa, semidecídua, e pela faixa de contato entre essas duas formações onde, também, ocorre o babaçu.

O município é cortado pelo rio Poti, o qual é servido pelos riachos Marimbas, Manilha, dos Cocos, Gameleira e do Brejo (CEPRO, 2008). A abertura de rodovias, bem como as atividades agropecuárias e de extrativismo do babaçu, foram responsáveis pela degradação da maior parte da vegetação original. As condições climáticas do município de Demerval Lobão, com altitude da sede a $112 \mathrm{~m}$, apresenta temperatura mínima de $22^{\circ} \mathrm{C} \mathrm{e}$ máxima de $28^{\circ} \mathrm{C}$, com clima Tropical Quente. A precipitação pluviométrica média anual (com registro de $1.800 \mathrm{~mm}$, na sede do município) é definida no Regime Equatorial Continental, com isoietas anuais entre 800 a $1.400 \mathrm{~mm}$, cerca de cinco a seis meses como os mais chuvosos, sendo o período restante do ano de estação seca. Os meses de janeiro, fevereiro e março correspondem ao trimestre mais chuvoso (CEPRO, 2008).

\section{Coleta de dados}

Os dados foram obtidos no período de abril/ 2007 a julho/2008, com visitas periódicas às 21 residências que possuíam quintais típicos e ativos (mantidos com trabalho familiar e tecnologia tradicional).

A coleta de material botânico foi realizada em quintais de nove localidades rurais com vegetação de cerrado, Chapadinha Sul, Carnaíba, Pontão, Buriti, Projeto Olho d'água, Sono, Alto da Boa Vista, São Gonçalo e Olho d'água (Figura 1).

Entrevistas foram realizadas, utilizando-se questionários padronizados (com 21 mantenedores dos quintais que apresentaram maior domínio cognitivo sobre os elementos). A amostra compreendeu 15 mulheres e seis homens, com idade variando entre 20 e 89 anos. Para a coleta de informações utilizouse a observação direta e entrevistas semiestruturadas com auxílio de gravador e diário de campo para informações adicionais. Concomitantemente, utilizou-se a técnica da turnê-guiada, na qual o mantenedor foi convidado a fazer caminhada pelo quintal durante a entrevista, fornecendo informações

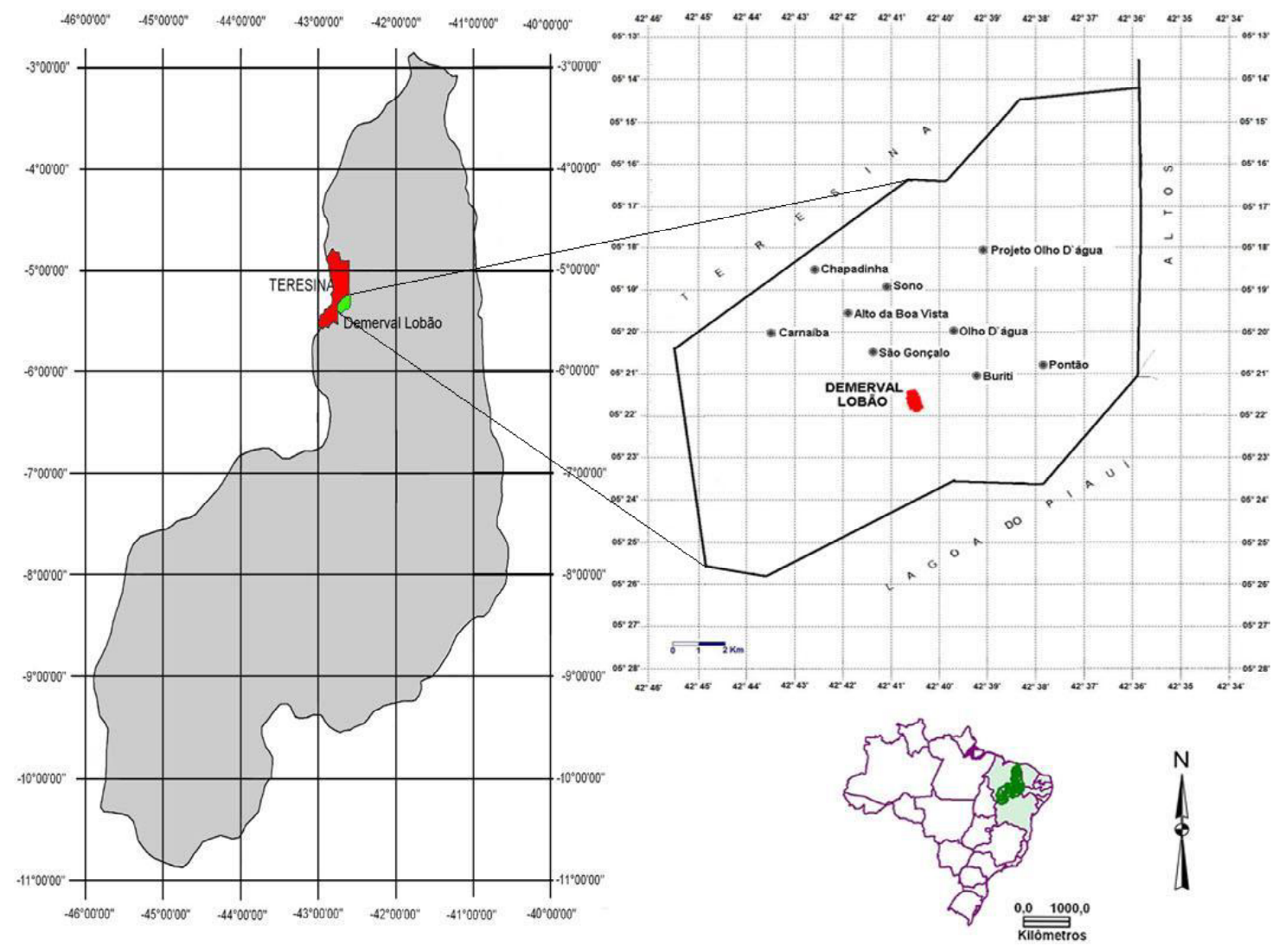

FIGURA 1. Localização do município de Demerval Lobão, Piauí, Brasil, destacando as áreas rurais estudadas. Fonte: Elaborado por Alexandre Nojoza. 
específicas sobre as plantas medicinais presentes (Albuquerque \& Lucena, 2004).

Seguiu-se Mori et al. (1989) para a realização das coletas e herborização do material botânico. As exsicatas encontram-se no acervo do Herbário Graziela Barroso (TEPB) da Universidade Federal do Piauí. As identificações das espécies foram feitas com base em bibliografia especializada, comparações com exsicatas identificadas, e envio a especialistas. O sistema de classificação adotado foi o de Cronquist (1981), com exceção da família Leguminosae, que obedeceu a Judd et al. (1999). As abreviaturas dos nomes dos autores das espécies estão de acordo com Brummitt \& Powell (1992) ou com o site IPNI (2008).

\section{Análise dos dados}

Com base na Organização Mundial de Saúde (OMS, 2000) e algumas modificações, as doenças foram agrupadas em 13 categorias, ou seja, transtornos do sistema respiratório; transtornos do sistema circulatório; transtornos do sistema nervoso; transtornos do sistema digestivo; transtornos do sistema genito-urinário; doenças do sistema osteomuscular; inflamações e dores em geral; doenças parasitárias; neoplasias; doenças do sangue; doenças da pele e do tecido celular subcutâneo; doenças das glândulas endócrinas e do metabolismo, e transtorno do sistema sensorial (visão).

Os Índices de Concordância de Uso foram calculados de acordo com Amorozo \& Gely (1988). A porcentagem de concordância quanto aos usos principais para cada espécie (CUP) mostra a importância relativa das plantas utilizadas nestas comunidades. Para o parâmetro CUP foram consideradas as espécies citadas por cinco ou mais informantes. Para isso, foram realizados os cálculos para cada espécie, onde CUP $=(I C U P /$ ICUE x 100) sendo ICUP = número de informantes citando o uso principal da espécie e ICUE = número total de informantes citando uso da espécie.

Calculou-se o Fator de Correção (FC) para cada espécie, o qual permite a extração de valores de importância relativos à espécie mais citada pelos informantes (CUPc).

$$
F C=I C U E / I C E M C, C U P=C U P \times F C
$$

onde: ICEMC = número de informantes que citaram a espécie mais citada.

\section{RESULTADO E DISCUSSÃO}

\author{
Dados sócio-econômicos dos moradores \\ da zona rural de Demerval Lobão \\ Quanto ao grau de instrução dos informantes \\ entrevistados, $9 \%$ são não escolarizados; $58 \%$
}

concluíram o ensino fundamental; $19 \%$ concluíram o ensino médio e $14 \%$ abandonaram os estudos.

$\mathrm{Na} z o n a$ rural do município de Demerval Lobão as pessoas trabalham principalmente nas atividades agropecuárias prestando serviços temporários, sobrevivendo da agricultura de subsistência, e limitados pela ausência de tecnologia e de mão-de-obra. A produção é quase que totalmente destinada ao consumo familiar. A renda mensal não ultrapassa um salário mínimo.

Entre os moradores entrevistados $72 \%$ são mulheres, as quais dedicam grande parte do tempo em contato direto com o quintal, produzindo e beneficiando alimentos e remédios para a família e, também, gerando excedentes para comercialização.

O quintal é visto pelos entrevistados como espaço apropriado para a realização de várias atividades, sejam relacionadas ao plantio, lazer, cultura, descanso e, em alguns casos, à criação de animais. Afirmação corroborada por Nunes (1994), que comenta que o quintal é o espaço em que o ser humano desenvolve as primeiras relações com o ambiente, sendo neste onde se brinca, relaciona-se, planta-se hortas, jardins, plantas medicinais, aprendendo assim a conviver de modo harmonioso com a diversidade ali existente.

\section{Dados botânicos e ecológicos}

Os moradores da zona rural do município de Demerval Lobão fazem uso de grupo diversificado de plantas presentes ao redor das casas, que se encontra distribuído em 100 espécies, 80 gêneros e 49 famílias (Tabela 1). As famílias melhor representadas foram Leguminosae (18 espécies), Euphorbiaceae (9) e Lamiaceae (7). Vieira et al. (2008), estudando as plantas medicinais na Comunidade Quilombola dos Macacos em São Miguel do Tapuio/PI e Almeida \& Albuquerque (2002), no levantamento de uso e conservação de plantas e animais medicinais no agreste Pernambucano, também incluíram estas famílias como as de maior representatividade. Franco \& Barros (2006) citaram as famílias Leguminosae e Euphorbiaceae, como as mais representativas no quilombo Olho d'Água dos Pires, Esperantina/PI.

Dentre as espécies citadas, a maioria apresenta hábito herbáceo $(47 \%)$, seguido de arbustivo (34\%) e arbóreo (19\%), observando pequena predominância de plantas cultivadas $(56 \%)$ em relação às nativas $(49 \%)$. Silva-Almeida \& Amorozo (1998) também constataram, em Rio Claro/SP, a predominância de espécies de porte herbáceo, e das plantas cultivadas.

As plantas medicinais de porte herbáceo são cultivadas geralmente em áreas mais restritas,

Rev. Bras. PI. Med., Botucatu, v.14, n.3, p.419-434, 2012. 


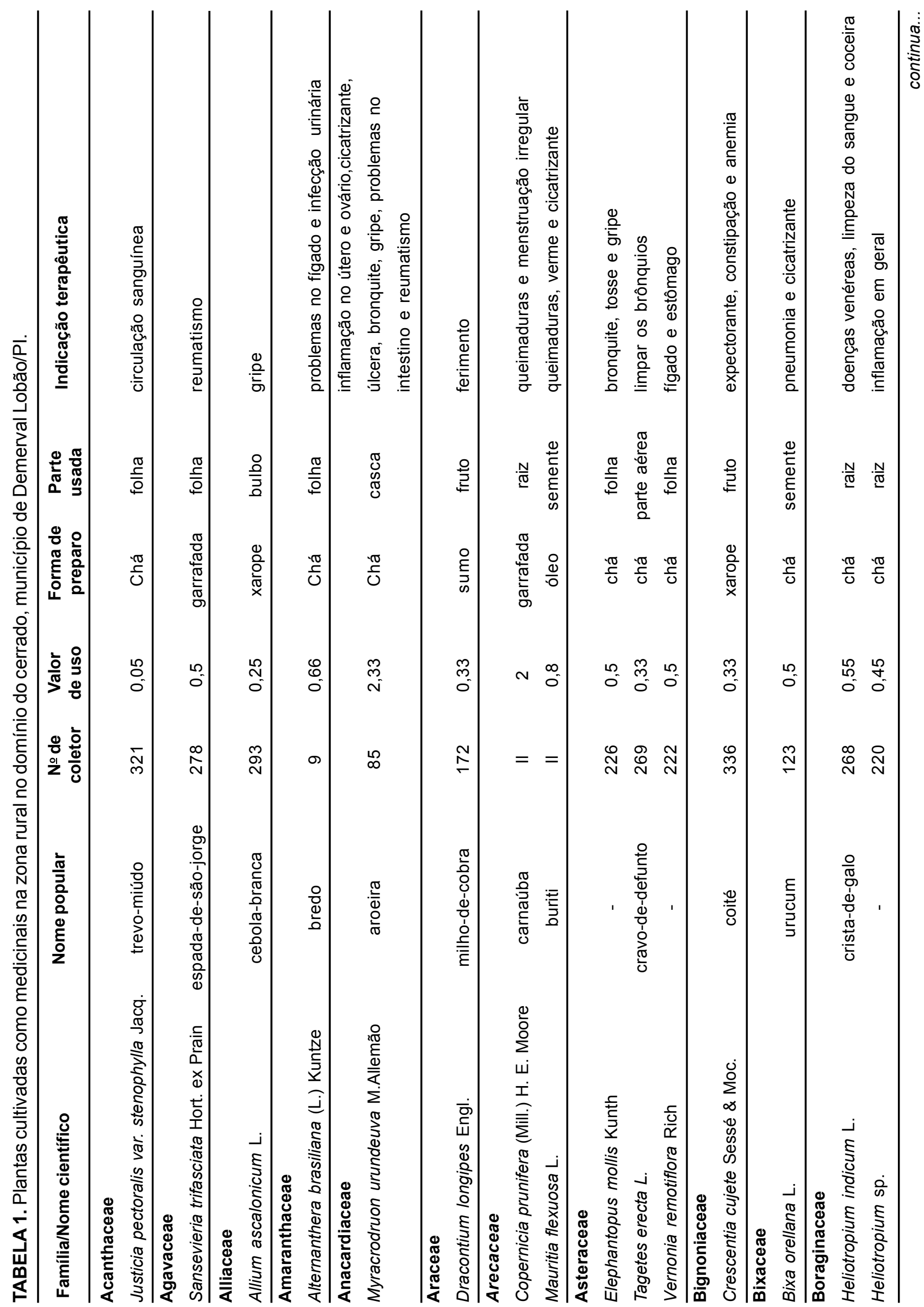

Rev. Bras. PI. Med., Botucatu, v.14, n.3, p.419-434, 2012. 


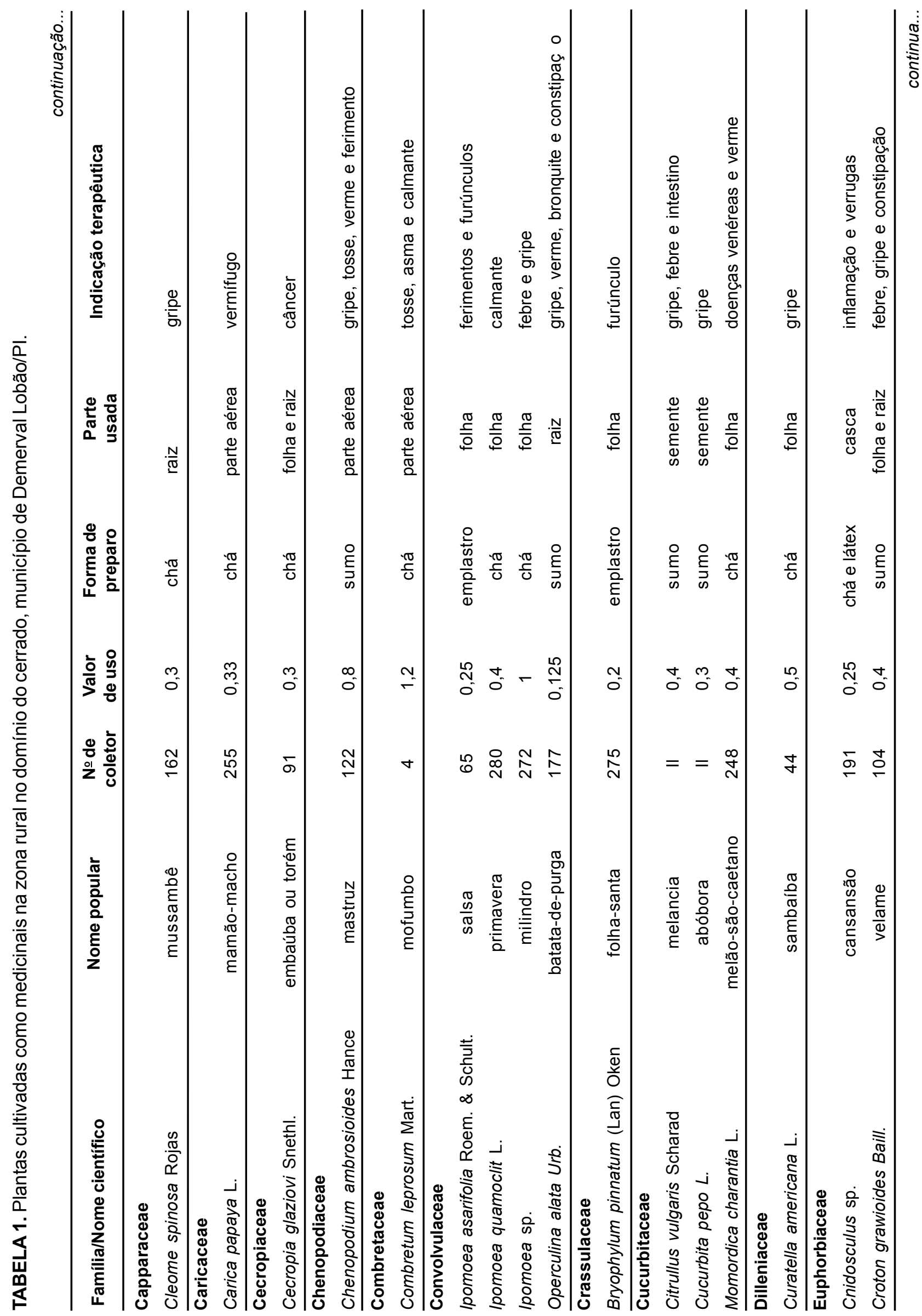

Rev. Bras. PI. Med., Botucatu, v.14, n.3, p.419-434, 2012. 


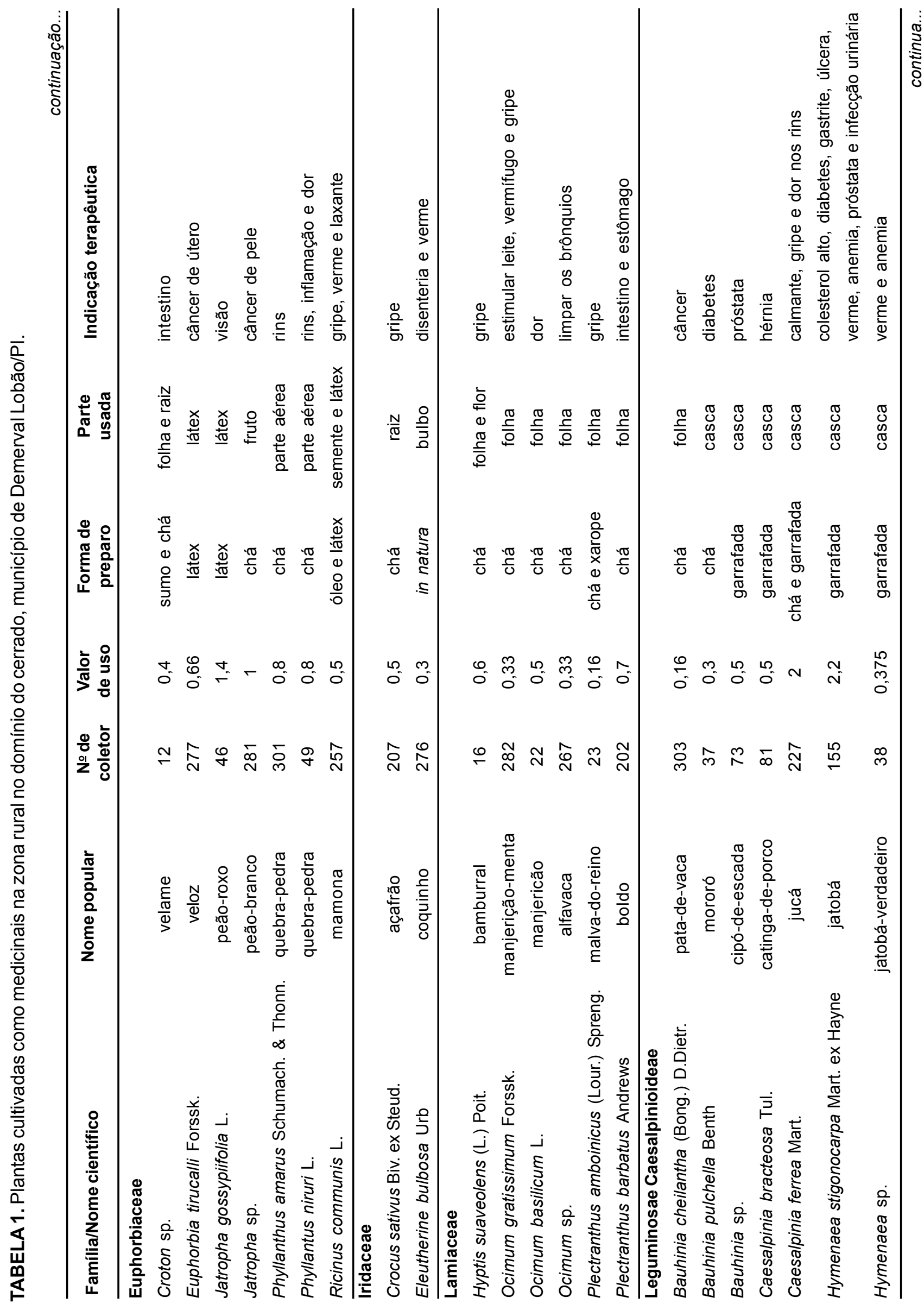

Rev. Bras. Pl. Med., Botucatu, v.14, n.3, p.419-434, 2012. 


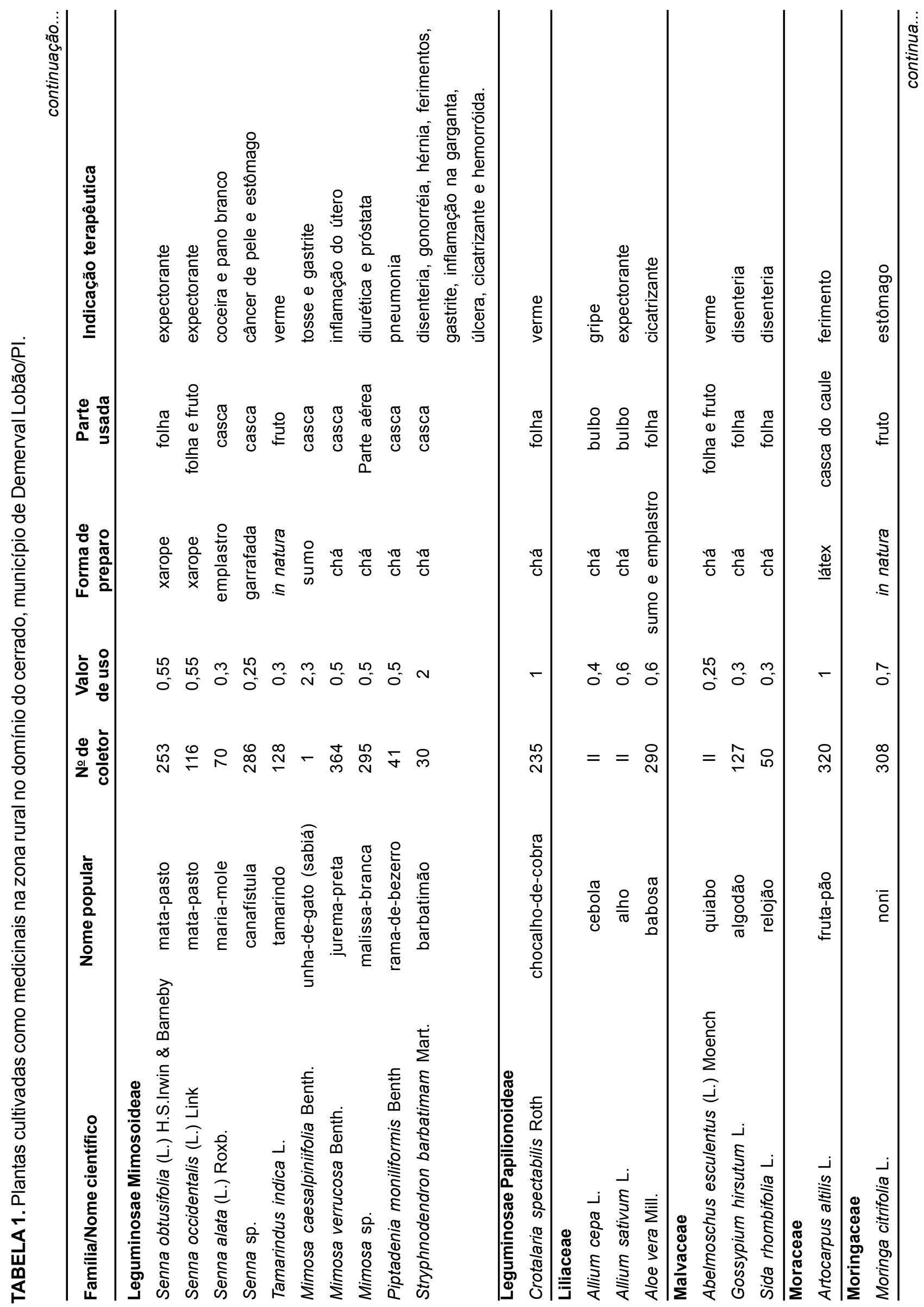

Rev. Bras. PI. Med., Botucatu, v.14, n.3, p.419-434, 2012. 


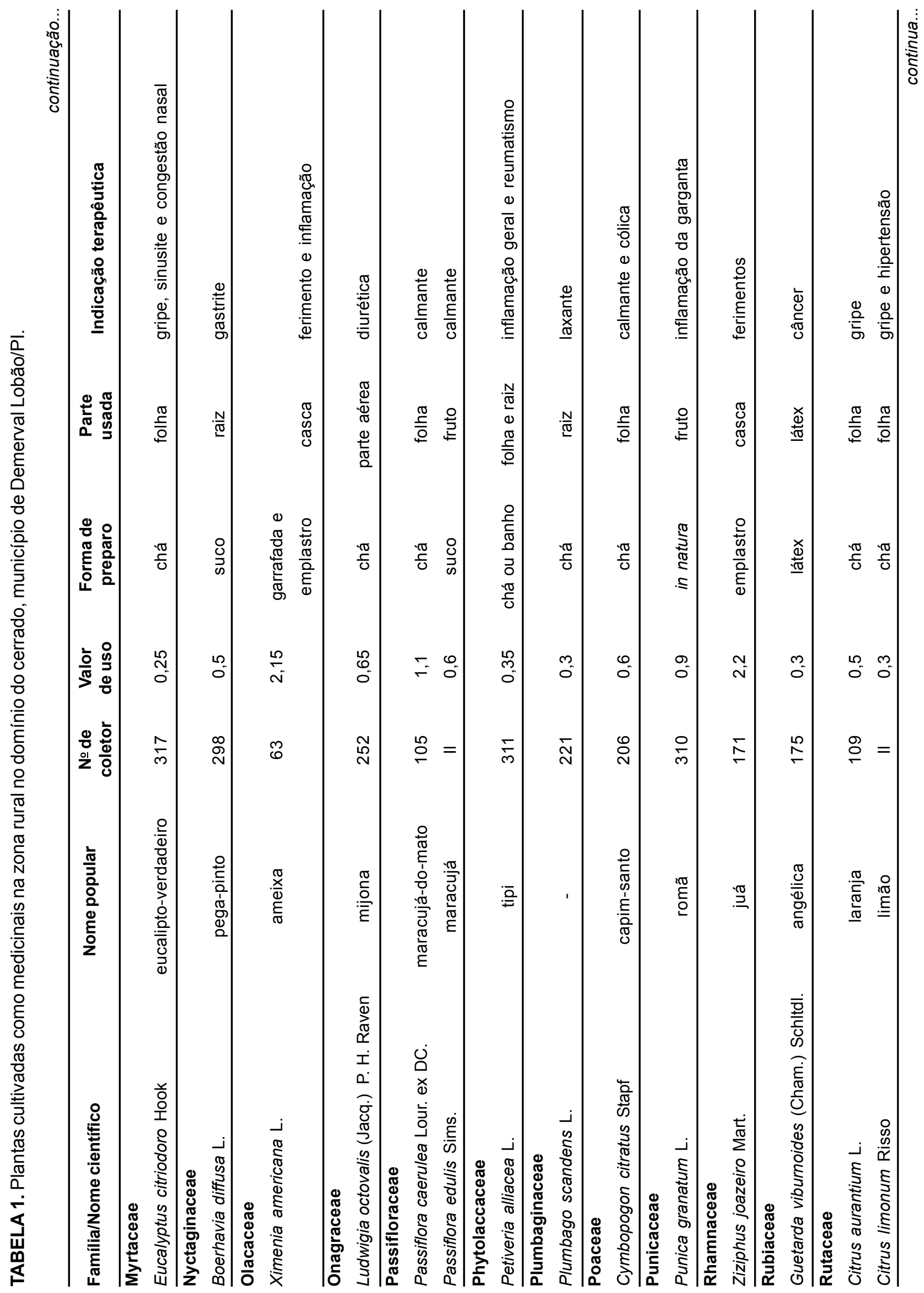

Rev. Bras. PI. Med., Botucatu, v.14, n.3, p.419-434, 2012. 


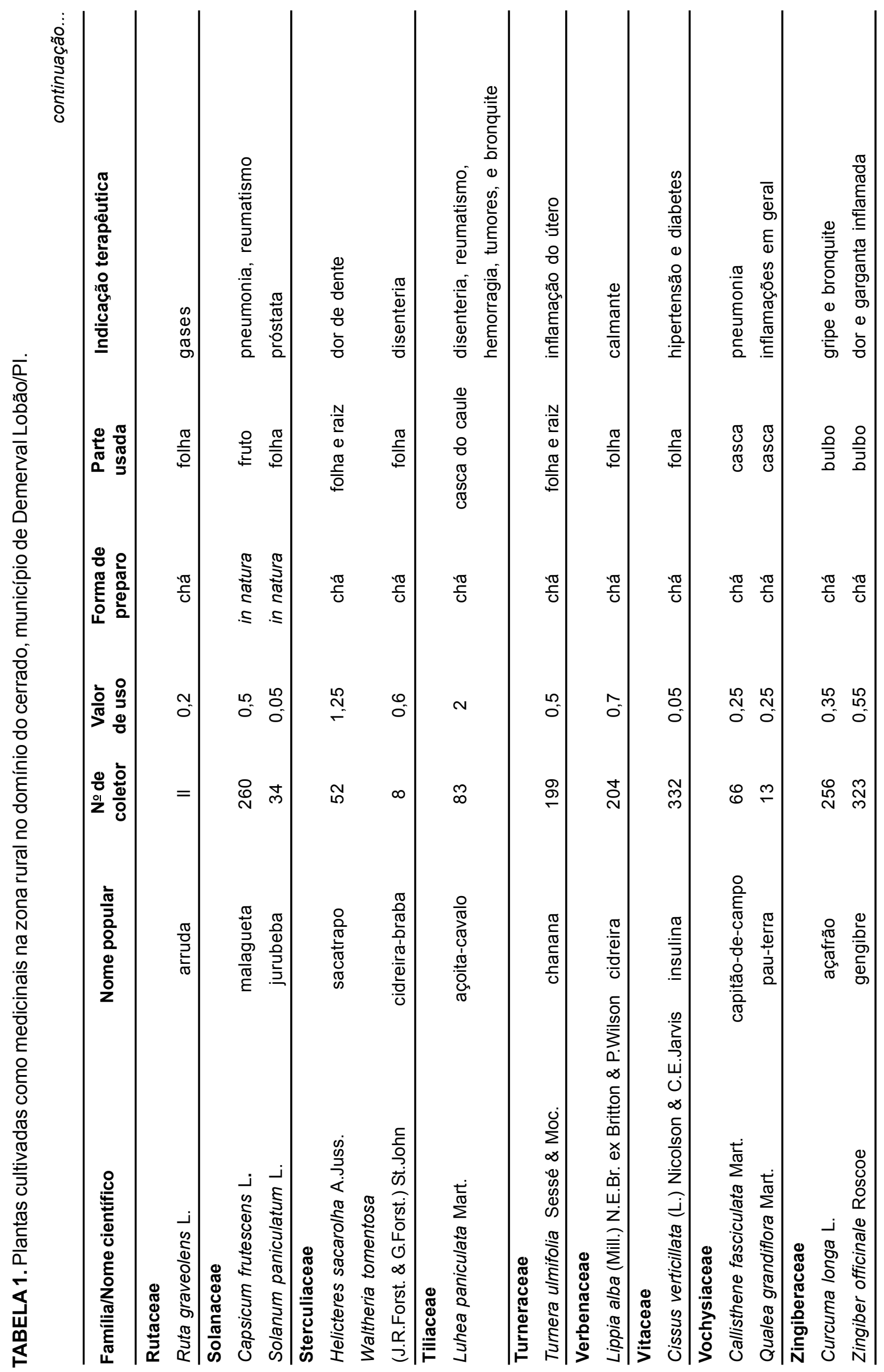

Rev. Bras. PI. Med., Botucatu, v.14, n.3, p.419-434, 2012. 
próximas à cozinha, pois facilita os cuidados uma vez que a mulher é a principal responsável por essas espécies. Essa situação é comum em quintais de outras regiões como relatado por Lamont et al. (1999); Murrieta \& WinklerPrins (2003) e Wezel \& Bender (2003).

Quanto a indicação de uso, constatou-se certo equilíbrio, pois $57 \%$ das espécies foram citadas pelos moradores para apenas um uso e $43 \%$ citadas para dois ou mais usos. Dados semelhantes foram obtidos por Franco \& Barros (2006) em Esperantina/ PI e Vieira (2008) em São Miguel do Tapuio/PI.

Quanto aos sistemas corporais tratados com as plantas medicinais o que obteve maior destaque foi o sistema respiratório (33\% das espécies), seguido pelo sistema digestivo (24\%) (Tabela 2). O maior número de espécies (20) foi indicado para o tratamento de gripes, podendo inferir que a grande quantidade de plantas empregadas para solucionar tal enfermidade parece estar relacionada às queimadas, à baixa umidade relativa do ar, e aos períodos de estiagem, os quais favorecem o surgimento de doenças relativas ao aparelho respiratório. Trabalhos realizados no estado de Pernambuco (Almeida \& Albuquerque, 2002) e no Mato Grosso (Amorozo, 2002) registraram também maior número de citações para transtornos do aparelho digestivo e respiratório.

Quanto à parte usada da planta no preparo dos remédios, destacam-se as folhas (34\%), casca do caule (19\%), e frutos e raízes (8\%) (Figura 2 ). Encontram-se dados semelhantes em Amorozo (2002) e Coelho et al. (2005) que também citaram as folhas como a parte mais usada nas comunidades de Tanquinho/BA e Mumbuca no Jalapão/TO, enquanto Albuquerque \&Andrade (2002) citam as cascas como preferidas por comunidades em áreas de caatinga no estado de Pernambuco, por estarem disponíveis o ano todo.

A forma de preparo dessas espécies é diversificada. O chá por decocção foi a forma de preparo mais indicada dentre as medicinais, com 58\% das indicações, seguida pela garrafada e sumo (7\% cada), emplastro, in natura e látex (6\% cada), xarope $(5 \%)$, óleo e suco ( $2 \%$ cada) e banho (1\%) (Figura 3$)$.

Quanto à posologia não foi observado rigor na quantidade a ser administrada. A maioria dos informantes não associa eventuais contra-indicações ao uso de remédios caseiros. De modo geral, a dosagem não necessita ser administrada com rigorosa exatidão, porém, Martins et al. (2000) citam que muitas substâncias podem ser tóxicas se a dosagem for exagerada.

Dados da utilização medicinal das plantas Sobressaíram-se algumas espécies com maior número de usos terapêuticos citados, tais como a Myracrodruon urundeuva, que é utilizada contra inflamação no útero e ovário, úlcera, bronquite, gripe, problemas no intestino, reumatismo, e como cicatrizante; a Hymenaea stigonocarpa, usada no tratamento do colesterol alto, diabetes, gastrite, úlcera, anemia, próstata, infecção urinária, e como vermífuga; a Stryphnodendron barbatiman, contra disenteria, gonorréia, hérnia, ferimentos, gastrite,

TABELA 2. Número de espécies vegetais e citações indicadas para cada categoria de doença (OMS, 2000), na zona rural do município de Demerval Lobão/PI.

\begin{tabular}{lcc}
\hline Categorias & $\begin{array}{c}\text { Número de } \\
\text { espécies vegetais }\end{array}$ & $\begin{array}{c}\text { Número de } \\
\text { citações reportadas }\end{array}$ \\
\hline Neoplasias & 6 & 13 \\
Transtornos do sistema digestivo & 24 & 55 \\
Doenças parasitárias & 14 & 17 \\
Transtornos do sistema nervoso & 7 & 18 \\
Transtornos do sistema genito-urinário & 13 & 28 \\
Transtornos do sistema respiratório & 33 & 76 \\
Transtornos do sistema circulatório & 3 & 8 \\
Doenças do sistema osteomuscular & 5 & 14 \\
Doenças das glândulas endócrinas & & 16 \\
e do metabolismo & 10 & 38 \\
Inflamações em geral & 17 & 28 \\
Doenças da pele & 13 & 8 \\
Doenças do sangue & 5 & 2 \\
Transtornos do sistema sensorial & 1 & \\
\hline
\end{tabular}

Rev. Bras. PI. Med., Botucatu, v.14, n.3, p.419-434, 2012. 


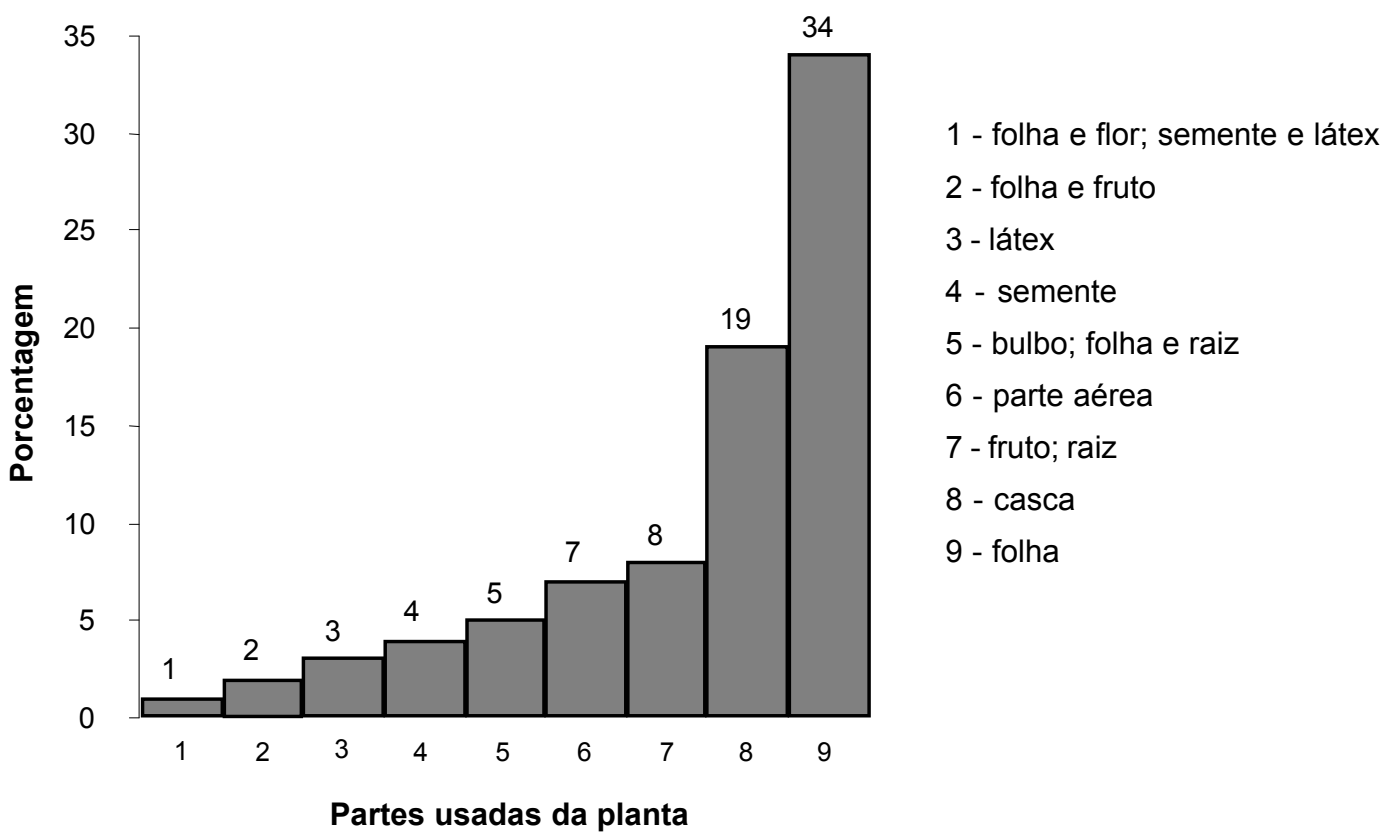

FIGURA 2. Percentual da parte da planta utilizada nos quintais de comunidades rurais do município de Demerval Lobão/PI.

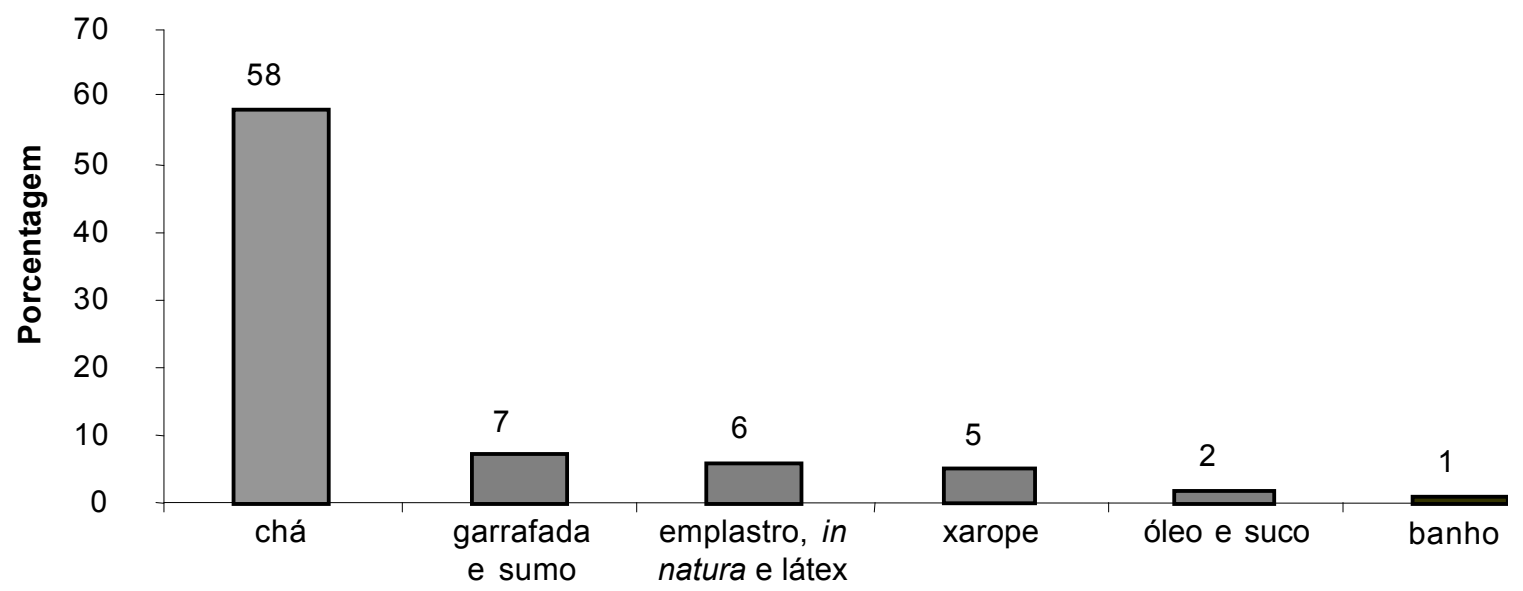

FIGURA 3. Distribuição percentual das formas de preparo das espécies medicinais utilizadas pelas comunidades rurais do município de Demerval Lobão/PI.

inflamação na garganta, úlcera, cicatrizante e hemorroida; e a Luhea paniculata no tratamento da disenteria, reumatismo, hemorragia, tumores e bronquite.

Outras espécies se destacaram pelo elevado número de citações pelos mantenedores, tais como a Ximenia americana, com propriedades anti-inflamatória e cicatrizante; a Chenopodium ambrosioides no combate à gripe, tosse, verme e ferimentos; a Phyllanthus amarus, no tratamento de doenças renais, inflamações e dores; a Plectranthus barbatus, bastante utilizada no tratamento de doenças do intestino e estômago; a Operculina alata, para tratar gripe, verme, bronquite e constipação; a Cymbopogon citratus, como calmante e contra cólica; a Punica granatum, no combate à inflamação na garganta; a Mentha sp, no combate a cólicas e vermes; a Lippia alba, como calmante e a Capsicum frutescens contra pneumonia e reumatismo. Esta última também é citada por Sablayrolles (2004), quanto à utilização pela população ribeirinha de Aveiro/PA para tratar a pneumonia.

$X$. americana e $M$. urundeuva também estão entre as mais citadas nos trabalhos de Chaves \& Barros (2008), Santos et al. (2008) e por Vieira et al. (2008).

Das espécies coletadas, observou-se 
pequena predominância de espécies exóticas (51\%) em comparação com as nativas (49\%). Nesse estudo, algumas das mais citadas para a cura de várias doenças foram o boldo ( $P$. barbatus), o capim-santo (C. citratus), a hortelã (Mentha $\mathrm{sp})$ e a romã $(P$. granatum), todas originárias do Velho Mundo, sendo o boldo nativo das regiões tropicais e úmidas da África e Ásia, a hortelã nativa da Europa e Ásia e a romã da Ásia. Esses dados assemelham-se aos de Pilla et al. (2006).

\section{Validação das Plantas Medicinais}

De acordo com Lorenzi \& Matos (2002), planta medicinal só é medicamento quando usada corretamente, o que só acontece quando o princípio ativo é identificado e evidenciado farmacologicamente. Nas comunidades estudadas algumas plantas são utilizadas como medicinais sem que as pessoas tenham eficiência comprovada, podendo muitas vezes lhes causar sérios danos, os quais muitas vezes passam despercebidos por se manifestarem em longo prazo. É o caso, por exemplo, de algumas Leguminosas conhecidas como cascaveleiras (Crotalaria $\mathrm{sp}$ ) citadas nesse estudo pela população para tratar verminoses, as quais contêm alto teor de alcalóides pirrolizidínicos. Todas elas devem ter o uso proibido para consumo caseiro especialmente quanto à ingestão das partes e extratos.

Estes alcalóides sendo ingeridos frequentemente, mesmo aos poucos, podem provocar lesões no fígado que resultam, após alguns anos, em disfunção hepática progressiva fatal. Um outro exemplo é o da romã (Punica granatum L.) que contém o alcalóide chamado peletierina, esta espécie foi indicada pela população para tratar inflamações na garganta, no entanto, Lorenzi \& Matos (2002) afirmam que esta pode causar intoxicações envolvendo náuseas, salivação excessiva, vômitos, dor abdominal, diarréia, confusão mental e, nas formas mais agudas, hipotensão seguida de colapso e morte.

No entanto, alguns estudos obtiveram sucesso graças às avaliações realizadas sob os auspícios da CEME (Central de Medicamentos do Ministério da Saúde) que incluíram dezenas de espécies mais usadas popularmente. Entre elas estão o capim santo (Cymbopogon citratus), o quebra-pedra (Phyllanthus niruri e outras espécies do mesmo gênero), o mastruz (Chenopodium ambrosioides), a embaúba (Cecropia glaziovii) e várias outras muito usadas no país.

\section{Etnobotânica quantitativa}

Segundo Friedman et al. (1986), uma planta com índice de concordância relativamente alto, isto é, que tenha vários informantes concordando com o mesmo uso terapêutico, talvez possa sugerir a real efetividade no tratamento da doença.

Para verificar a importância relativa das plantas utilizadas quanto ao número de informantes que citaram e a concordância dos usos citados, foram listadas plantas mencionadas por cinco ou mais informantes, totalizando 30 plantas (Tabela 3). Várias plantas apresentaram forte consistência de uso (CUP de $100 \%$ ), como Allium cepa, A. sativum, Aloe vera, Cnidosculus sp, Crotalaria spectabilis, Euphorbia tirucalli, Lippia alba, Mimosa caesalpiniifolia, M. verrucosa, Plectranthus barbatus, Punica granatum, Qualea grandiflora, Senna occidentalis e Turnera ulmifolia. Porém, quando se aplica o valor de correção, apenas uma ( $P$. barbatus) obteve o valor mais alto $(1,00)$, comparando-se com a espécie mais citada; o que mostra que o conhecimento não se aplica a todos os informantes. Apenas quatro das trinta espécies mais citadas apresentaram CUPc acima de 60\% (Cnidosculus sp, L. alba, M. verrucosa e P. barbatus).

Plectranthus barbatus foi a mais citada e também a que apresentou os índices mais altos de CUP $(100 \%)$, FC $(1,00)$ e CUPc $(100 \%)$ para dores no estômago e problemas do fígado. Esses dados assemelham-se aos apresentados por Pilla et al. (2006). Para Pinto et al. (2006), Chenopodium ambrosioides foi a que obteve valor de CUP mais alto dentre as plantas medicinais utilizadas nas comunidades rurais da mata atlântica-Itacaré/BA.

$P$. barbatus tem os efeitos comprovados por testes experimentais, de acordo com o ensaio farmacológico realizado por Fischman et al. (1991); o extrato aquoso das folhas apresentou ação hipossecretora gástrica, ajudando a diminuir, não só o volume de suco gástrico, como também a acidez.

\section{CONCLUSÃO}

As espécies existentes nos 21 quintais analisados suprem uma série de necessidades básicas dos moradores e suas famílias, contudo, a finalidade principal dos quintais da zona rural de Demerval Lobão é o de promover a complementação alimentar das unidades familiares e cultivar espécies medicinais que contribuam para a saúde da família.

O universo de plantas indicadas como importantes para a população e cultivadas nos quintais apresentam alta diversidade e baixa densidade por espécie, concluindo-se que constituem um importante espaço para a conservação da diversidade biológica e sócio-cultural dessas populações.

A tradicional transmissão de conhecimentos dos pais para os filhos foi a mais representativa forma de aquisição de informações acerca das plantas medicinais.

Ao se resgatar os conhecimentos terapêuticos locais, pode-se fornecer relevantes contribuições para 
TABELA 3. Porcentagem de concordância das espécies vegetais quanto ao(s) uso(s) principal(is) (espécies citadas por cinco ou mais informantes). ICUE - número de informantes citando uso da espécie; ICUP - número de informantes citando uso principal; CUP - índice de concordância de uso; FC - fator de correção; CUPc - CUP corrigida.

\begin{tabular}{|c|c|c|c|c|c|c|c|}
\hline Espécie & UP & ICUE & ICUP & CUP & FC & CUPc & ICEMC \\
\hline Allium sativum $\mathrm{L}$. & expectorante & 8 & 8 & 100,0 & 0,47 & 47,1 & 17 \\
\hline Allium cepa L. & Gripe & 7 & 7 & 100,0 & 0,41 & 41,2 & 17 \\
\hline Aloe vera Mill. & cicatrizante & 9 & 9 & 100,0 & 0,53 & 52,9 & 17 \\
\hline Bixa orellana L. & Pneumonia & 12 & 10 & 83,3 & 0,71 & 58,8 & 17 \\
\hline Carica papaya L. & Vermífugo & 10 & 10 & 100,0 & 0,59 & 58,8 & 17 \\
\hline Chenopodium ambrosioides Hance & gripe, tosse e vermífugo & 14 & 13 & 92,9 & 0,82 & 76,5 & 17 \\
\hline Citrus aurantium L. & Gripe & 6 & 5 & 83,3 & 0,35 & 29,4 & 17 \\
\hline Citrus limonum Risso & Gripe & 8 & 6 & 75,0 & 0,47 & 35,3 & 17 \\
\hline Cnidosculus sp & inflamação e verrugas & 12 & 12 & 100,0 & 0,71 & 70,6 & 17 \\
\hline Combretum leprosum Mart. & tosse e asma & 7 & 4 & 57,1 & 0,41 & 23,5 & 17 \\
\hline Crescentia cujete Sessé \& Moc. & expectorante e laxante & 6 & 5 & 83,3 & 0,35 & 29,4 & 17 \\
\hline Crotalaria spectabilis Roth & Vermífugo & 5 & 5 & 100,0 & 0,29 & 29,4 & 17 \\
\hline Cymbopogon citratus Stapf & Cólica & 9 & 7 & 77,8 & 0,53 & 41,2 & 17 \\
\hline Euphorbia tirucalli Forssk. & câncer de útero & 6 & 6 & 100,0 & 0,35 & 35,3 & 17 \\
\hline $\begin{array}{l}\text { Hymenaea stigonocarpa Mart. ex Hayne } \\
\text { Lippia alba (Mill.) N.E.Br. }\end{array}$ & colesterol alto e diabetes & 7 & 3 & 42,9 & 0,41 & 17,6 & 17 \\
\hline ex Britton \& P.Wilson & Calmante & 15 & 15 & 100,0 & 0,88 & 88,2 & 17 \\
\hline Mimosa caesalpiniifolia Benth. & tosse e gastrite & 7 & 7 & 100,0 & 0,41 & 41,2 & 17 \\
\hline Mimosa verrucosa $\mathrm{L}$. & inflamação do útero & 12 & 12 & 100,0 & 0,71 & 70,6 & 17 \\
\hline Momordica charantia L. & $\begin{array}{l}\text { Vermífugo } \\
\text { inflamação no útero e }\end{array}$ & 11 & 9 & 81,8 & 0,65 & 52,9 & 17 \\
\hline Myracrodruon urundeuva M.Allemão & $\begin{array}{l}\text { ovário, afecções cutâneas } \\
\text { e úlcera }\end{array}$ & 16 & 14 & 87,5 & 0,94 & 82,4 & 17 \\
\hline Operculina alata Urb. & vermífugo e laxante & 9 & 8 & 88,9 & 0,53 & 47,1 & 17 \\
\hline Phyllantus niruri L. & Rins & 16 & 14 & 87,5 & 0,94 & 82,4 & 17 \\
\hline Plectranthus barbatus Andrews & estômago e fígado & 17 & 17 & 100,0 & 1,00 & 100,0 & 17 \\
\hline Punica granatum $\mathrm{L}$. & inflamação da garganta & 6 & 6 & 100,0 & 0,35 & 35,3 & 17 \\
\hline Qualea grandiflora Mart. & inflamações em geral & 6 & 6 & 100,0 & 0,35 & 35,3 & 17 \\
\hline Ricinus communis $\mathrm{L}$. & vermífugo e laxante & 13 & 10 & 76,9 & 0,76 & 58,8 & 17 \\
\hline Senna occidentalis (L.) Link & expectorante & 6 & 6 & 100,0 & 0,35 & 35,3 & 17 \\
\hline Stryphnodendron barbatiman Mart. & $\begin{array}{l}\text { ferimentos, gastrite, } \\
\text { inflamação na garganta, } \\
\text { e cicatrizante }\end{array}$ & 14 & 9 & 64,3 & 0,82 & 52,9 & 17 \\
\hline Turnera ulmifolia Sessé \& Moc. & inflamação do útero & 6 & 6 & 100,0 & 0,35 & 35,3 & 17 \\
\hline Ximenia americana L. & Inflamação & 14 & 12 & 85,7 & 0,82 & 70,6 & 17 \\
\hline
\end{tabular}

a conservação da diversidade biológica e do rico acervo cultural concernente às práticas extrativistas e ao manejo de recursos naturais.

Com esse estudo pôde-se constatar que os quintais são espaços mutantes e complementares às demais unidades produtivas da propriedade onde os moradores investem numa biodiversidade útil, seja ela cultivada ou nativa, constituindo-se também locais de teste e seleção e de acúmulo de conhecimentos sobre o uso de plantas. 


\section{REFERÊNCIA}

ALBUQUERQUE, U.P.; ANDRADE, L.H.C. Conhecimento botânico tradicional e conservação em uma área de caatinga no Estado de Pernambuco, Nordeste do Brasil. Acta Botanica Brasilica, n.16, p.273-85, 2002.

ALBUQUERQUE, U.P.; LUCENA, R.F.P. Métodos e técnicas para a coleta de dados. In: ALBUQUERQUE, U.P.; LUCENA, R.F.P. (Orgs.). Métodos e técnicas na pesquisa etnobotânica. Recife: Livro Rápido/NUPEEA, 2004. p.56-72.

ALMEIDA, C.F.; ALBUQUERQUE, U.P. Uso e conservação de plantas e animais medicinais no estado de Pernambuco (Nordeste do Brasil): um estudo de caso. Interciência, v.27, n.6, p.276-85, 2002.

AMOROZO, M.C.M. A abordagem etnobotânica na pesquisa de plantas medicinais. In: DI STASI, L.C. (Org.). Plantas medicinais: arte e ciência. São Paulo: Unesp, 1996. p.29-32.

AMOROZO, M.C.M. Uso e diversidade de plantas medicinais em Santo Antônio do Leverger, MT, Brasil. Acta Botanica Brasilica, v.16, n.2, p.189-203, 2002. AMOROZO, M.C.M.; GÉLY, A. Uso de plantas medicinais pelos caboclos do baixo Amazonas, Barcarena, Pará, Brasil. Boletim do Museu Paraense Emílio Goeldi, n.4, p.47-131, 1988.

ARNOUS, A.H.; SANTOS, A.S.; BEINNER, R.P.C. Plantas medicinais de uso caseiro: conhecimento popular e interesse por cultivo comunitário. Espaço para a Saúde, n.6, p.1-6, 2005.

BERG, M.E.; SILVA, M.H.L. Contribuição ao conhecimento da flora medicinal do Piauí. In: REUNIÃO NORDESTINA DE BOTÂNICA, 1985, 8., Recife. Anais ... Recife: Sociedade Botânica do Brasil - Seccional Pernambuco, 1985, p.151-64.

BRUMMITT, R.K.; POWELL, C.E. Author of plant names. London: Royal Botanic Gardens Key, 1992. 732p.

CEPRO - Fundação Centro de Pesquisas Econômicas e Sociais. Teresina: visão global. 2.ed. 2008. 89p.

CHAVES, E.M.F.; BARROS, R.F.M. Non - timber forest products. Functional Ecossystems and Comunities, v.2, n.8, p.21-31, 2008.

COELHO, F.B.R. et al. Levantamento etnofarmacológico realizado na comunidade Mumbuca localizada no Jalapão - TO. Revista Eletrônica de Farmácia, v.2, n.2, p.52-5, 2005. Disponível em: <www.farmacia.ufg.br/ revista/vol2>. Acesso em: 15 jul. 2007.

CRONQUIST, A. An integrated system of classification of flowering plants. New York: Columbia Universaty Press, 1981. 1262p.

FISCHMAN, L.A. et al. The water extract of Coleus barbatus Benth: decrease gastric secretion in rats. Memórias do Instituto Oswaldo Cruz, v.86, n.2, p.141-3, 1991.

FRANCO, E.A.P.A.; BARROS, R.F.M. Uso e diversidade de plantas medicinais no Quilombo Olho D'água dos Pires, Esperantina, Piauí. Revista Brasileira de Plantas Medicinais, v. 8, n. 3, p.78-88, 2006.

FRIEDMAN, J. et al. A preliminery classification of the healing potential of medicinal plants, based on a rational analysis of an ethnopharmacological field survey among bedouins in the Negev desert, Israel. Journal of Ethnopharmacology, v.16, p.275-87, 1986.
GAZZANEO, L.R.S.; LUCENA, R.F.P.; ALBUQUERQUE, U.P. Knowledge and use of medicinal plants by local specialists in region of Atlantic Forest in the state of Pernambuco (Northeastern Brazil). Journal of Ethnobiology and Ethnomedicine, v.32, n.1, p.1-11, 2005. GUARIM-NETO, G.; MORAIS, R.G. Recursos medicinais de espécies do cerrado de Mato Grosso: um estudo bibliográfico. Acta Botanica Brasilica, v.17, n.4, p.56184, 2003

JACOBY, A. et al. Plantas medicinais utilizadas pela comunidade rural de Guaramirim, município de Irati, Paraná. Revista Ciências Exatas e Naturais, v.4, n.1, p.79-89, 2002.

IBGE. Censo 2008. Disponível em: <www.ibge.gov.br>. Acesso em: 05 set. 2008.

IPNI. International plant names index. Disponível em: $<w w w . i p n i . o r g>$. Acesso em 05 set. 2008.

JUDD, W.S. et al. Plant systematics: a phylogenetic approach. Massachusetts: Sinauer associates, 1999. $462 p$.

LAMONT, S.R.; ESHBAUGH, W.A.; GREENBERG, A.M. Composition, diversity, and use of homegardens among three Amazonian villages. Economic Botany, v.53, n.3, p.312-26, 1999.

LOK, R.; MENDEZ, E. El uso del ordenamiento local del espacio para una clasificación de huertos na Nicaragua. In: LOK, R. (Ed.). Huertos tradicionales de América Central: características, beneficios e importancia, desde um enfoque multidisciplinario. Turrialba: CATIE, 1998. p.129-49.

LORENZI, H.; MATOS, F.J.A. Plantas medicinais no Brasil: nativas e exóticas. Nova Odessa: Plantarum, 2002. $572 \mathrm{p}$

MARODIN, S.M.; BAPTISTA, L.R.M. Plantas medicinais do município de Dom Pedro de Alcântara, estado do Rio Grande do Sul: espécies, famílias e usos em três grupos de população humana. Revista Brasileira de Plantas Medicinais, v.6, n.3, p.1-9, 2002.

MARTINS, E.R. et al. Plantas medicinais. Viçosa: Editora UFV, 2000. 220p.

MEDEIROS, M.F.T.; FONSECA, V.S.; ANDREATA, R.H.P. Plantas medicinais e seus usos pelos sitiantes da Reserva Rio das Pedras, Mangaratiba, RJ, Brasil. Acta Botanica Brasilica, v.9, n.18, p.391-9, 2004.

MING, L.C. et al. Manejo e cultivo de plantas medicinais: algumas reflexões sobre as perspectivas $e$ necessidades no Brasil In: COELHO, M.F.B.; COSTA JÚNIOR, P.E.; DOMBROSKI, J.L.D. (Orgs.). Diversos olhares em Etnobiologia, Etnoecologia e Plantas Medicinais. Cuiabá: SBEE, 2002, 250p.

MORI, S.A. et al. Manual de manejo do herbário fanerogâmico. 2.ed. Ilhéus: CEPLAC, 1989. 104p. MURRIETA, R.S.S.; WINKLERPRINS, A.M.G.A. Flowers of water: homegardens and gender roles in a riverine caboclo community in the lower Amazon, Brazil. Culture and Agriculture, v.25, n.1, p.35-47, 2003.

NUNES, N. Ciência e Trópico. Recife: Editora Massangana, 1994, 383p.

OMS (Organização Mundial de Saúde). Classificação estatística internacional de doenças e problemas relacionados à saúde. São Paulo: EDUSP, 2000, 1056p. PASA, M.C.; SOARES, J.N. ; GUARIM-NETO, G. Estudo 
etnobotânico na comunidade de Conceição-Açu (alto da bacia do rio Aricá Açu, MT, Brasil). Acta Botanica Brasilica, v.17, n.19, p.195-207, 2005.

PILLA, M.A.C.; AMOROZO, M.C.M.; FURLAN, A. Obtenção e uso das plantas medicinais no distrito de Martim Francisco, município de Mogi-Mirim, SP, Brasil. Acta Botanica Brasilica, v.20, n.4, p.789-802, 2006.

PINTO, E.P.P.; AMOROZO, M.C.M.; FURLAN, A. Conhecimento popular sobre plantas medicinais em comunidades rurais de mata atlântica - Itacaré, BA, Brasil. Acta Botanica Brasilica, v.20, n.4, p.751-62, 2006.

SABLAYROLLES, M.G.P. Diversidade e uso de plantas em quintais ribeirinhos de Brasília Legal - Aveiro, Pará (Brasil). 2004. 158p. Tese (Doutorado - Área de concentração em Biologia Vegetal) - Departamento de Botânica, Universidade Federal de Pernambuco, Recife.

SANTOS, L.G.P. et al. Diversity of useful plant resources in the city of Monsenhor Gil, Piauí State, Brazil. Functional Ecossystems and Comunities, v.2, n.8, p.72-80, 2008.

SILVA, A.J.R.; ANDRADE, L.H.C. Etnobotânica nordestina: estudo comparativo da relação entre comunidades e vegetação na Zona do Litoral - Mata do Estado de Pernambuco, Brasil. Acta Botanica Brasilica, v.28, n.19, p.45-60, 2005.

SILVA-ALMEIDA, M.F.; AMOROZO, M.C.M. Medicina popular no Distrito de Rio Claro, Estado de São Paulo. Brazilian Journal of Ecology, v.6, n.2, p.36-46, 1998.

VIEIRA, F.J. et al. Quilombola of Macacos Community, São Miguel do Tapuio City, Piauí State: history, use and conservation of plant resources. Functional Ecossystems and Comunities, v.2, n.8, p.81-7, 2008. WEZEL, A.; BENDER, S. Plant species diversity of homegardens of Cuba and its significance for household food suply. Agroforestry Systems, v.57, n.1, p.39-49, 2003. 